\title{
Alois Stacher - † 20. Juli 2013
}

Alois Stacher wurde am 16.02.1925 in Wien geboren. Dort besuchte er die Volksschule und das humanistische Gymnasium und legte im Jahr 1943 die Reifeprüfung ab. Anschließend wurde er zur Deutschen Wehrmacht eingezogen und am 27.04.1945 schwerst verwundet. Nach einem mehr als 2-jährigen Aufenthalt in einem amerikanischen Lazarett kam er

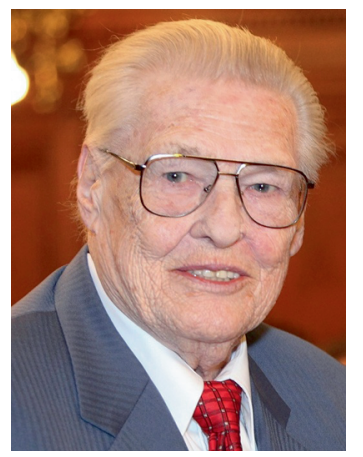
im Jahre 1947 nach Wien zurück, wo er noch im selben Jahr mit dem Medizinstudium begann. Das Studium schloss er am 15.07.1952 mit der Promotion zum Doktor der Humanmedizin ab. Seine erste Tätigkeit als Arzt begann er im Wiener HanuschKrankenhaus, das sein gesamtes ärztliches Berufsleben bis zum Ende seiner aktiven Tätigkeit prägte. Im Jahre 1959 erhielt er die Facharztanerkennung für Innere Medizin. In der Folge widmete sich Stacher vor allem der Hämatologie. Seine erfolgreiche wissenschaftliche Tätigkeit auf diesem Gebiet war die Grundlage für die Habilitation im Fach Innere Medizin und die Ernennung zum Titularprofessor im Jahre 1973. Von 1975 bis 1990 leitete er das hämatologisch-onkologische Zentrum am Hanusch-Krankenhaus sowie das Ludwig Boltzmann-Institut für Leukämieforschung und Hämatologie, das dem Krankenhaus von 1968 bis 1993 angegliedert war. Schwerpunkt der wissenschaftlichen Tätigkeit von Stacher war in dieser Zeit die Leukämie- und Lymphomforschung, mit der er sich auch international einen wissenschaftlichen Ruf erworben hat.

In der Bevölkerung Wiens war Stacher vor allem durch seine gesundheitspolitische Tätigkeit bekannt. Im Jahr 1973 wurde er zum amtsführenden Stadtrat für Gesundheit und Soziales der Stadt Wien gewählt und übte das Amt bis zum Ende des Jahres 1989 aus. Dabei konzentrierte er sich auf den Ausbau des Sozialwesens und die Reorganisation der Wiener Spitäler. In diesem Rahmen initiierte er auch eine international anerkannte Psychiatriereform. Wie dem Verfasser des vorliegenden Nachrufs aus persönlichen Gesprächen bekannt ist, hat das Gelingen dieser Reform sowie die Errichtung eines modernen Spitals mit der Bezeichnung «Sozialmedizinisches Zentrum Ost» (Donauspital) im 22. Wiener Gemeindebezirk Stacher mit Stolz und Befriedigung erfüllt.
Stacher hatte aber auch noch zahlreiche andere Funktionen inne, von denen nachfolgend nur die wichtigsten genannt werden sollen:

- Präsident des Landesverbandes Wien des Österreichischen Roten Kreuzes 1973-1989

- Präsident des «Medizinisch-Wissenschaftlichen Fonds des Bürgermeisters der Bundeshauptstadt Wien» 1978-1989

- Präsident des Kuratoriums für psychosoziale Dienste in Wien 1979-1989

- Gründer und Präsident des Wiener Krankenanstaltenverbandes 1975-1989

- Vizepräsident der Krankenhausberatungsagentur des Gesundheitsministeriums 1988-1989

- stellvertretender Vorsitzender des «Fonds Gesundes Österreich» 1974-1989

- Mitglied des Kuratoriums des «Kardinal Innitzer-Studienfonds» 1974-1989

- Mitglied des «Kuratoriums der Hochschuljubiläumsstiftung» 1976-1989

- Mitglied des Vereinsausschusses des «Rudolfiner-Vereins», der die älteste Krankenpflegeschule Europas vor der Schließung bewahrte.

Wer Stacher kannte weiß, dass er all diese Aufgaben nicht nur formal ausfüllte, sondern aktiv mitgestaltete und mit seiner Persönlichkeit prägte.

Angesichts seiner vielfältigen Verantwortungen und seines medizinischen Hintergrunds als streng naturwissenschaftlich orientierter Hämatoonkologe erscheint es besonders bemerkenswert, dass Stacher sich immer auch für die Belange einer ganzheitlichen Medizin interessiert und aktiv eingesetzt hat. Er betonte in persönlichen Gesprächen immer wieder, dass die Medizin während seines Studiums und zu Beginn seiner ärztlichen Tätigkeit zu einem erheblichen Anteil traditionelle Erfahrungsheilkunde war. Begriffe wie Komplementär- oder Alternativmedizin existierten zu dieser Zeit noch nicht, sondern entwickelten sich aus einer Situation, in der erkannt wurde, dass die sogenannte Schulmedizin nicht in der Lage war, alle gesundheitlichen Probleme zu lösen. Stacher wies immer wieder auf die etwas paradoxe Situation hin, dass einerseits die naturwissenschaftlich orientierte Medizin beeindruckende Fortschritte zum Wohl der Patienten verzeichnete, andererseits aber die Zufriedenheit der Menschen mit dem Gesund-

\section{KARGER}

Fax +497614520714

Information@Karger.com

www.karger.com
(C) 2013 S. Karger GmbH, Freiburg

Accessible online at:

www.karger.com/fok 
heitsangebot nicht proportional dazu anstieg. Dies waren die wichtigsten Beweggründe dafür, dass Stacher sich schon seit Beginn seiner ärztlichen Tätigkeit auch für Denkweisen und Methoden interessiert hat, die nicht ausschließlich der Schulmedizin zugeordnet werden können. Der erste Kontakt Stachers mit komplementärmedizinischen Methoden war die Neuraltherapie, der bald darauf die Akupunktur und die Homöopathie folgten. Eine weitere Motivation für die Auseinandersetzung mit diesen Methoden war sein Interesse an der Regulationsmedizin und der Matrix. Eine Folge seines Interesses an diesen Themen war die Gründung des «Wiener Teams», das von Namen wie Otto Bergsmann, Johannes Bischko, Franz Hopfer, Gottfried Kellner, Rudolf Maresch, Felix Perger und Alfred Pischinger geprägt war und Forschungsaktivitäten auf den genannten Gebieten entfaltet hat. Den Anreiz zur Gründung der «Wiener Internationalen Akademie für Ganzheitsmedizin» (GAMED) im Jahre 1988 gab ein von Stacher organisierter internationaler Kongress in Wien über Ganzheitsmedizin. Damit verfolgte er das Ziel, eine vorurteilslose Diskussion und den Dialog verschiedener Denkrichtungen $\mathrm{zu}$ fördern. Das grundlegende Anliegen der GAMED ist eine Verbindung zwischen naturwissenschaftlich fundierter universitärer Medizin und der Erfahrungsheilkunde unter Berücksichtigung der psychischen und geistigen Aspekte sowie komplexer und regulativer Prozesse. Stacher hat immer auf die Wichtigkeit der postgradualen Ausbildung und der wissenschaftlichen Tätigkeit hingewiesen. Er war auch Mitbegründer der Zeitschrift Forschende Komplementärmedizin, wodurch die Möglichkeit zur Publikation von Arbeiten auf dem Gebiet der Komplementärmedizin geschaffen wurde.

Die wissenschaftliche Tätigkeit Stachers manifestierte sich in der Herausgabe und Autorschaft von 26 wissenschaftlichen
Büchern und über 382 wissenschaftlichen Publikationen auf dem Gebiet der Inneren Medizin, vor allem im Bereich der Hämatologie sowie der Leukämie- und Lymphomforschung, aber auch im sozialen und gesundheitspolitischem Sektor. In den letzten Jahren seines Wirkens kamen noch Beiträge über Ganzheitsmedizin hinzu. Von den zahlreichen Ehrungen und Auszeichnungen, die Stacher zuteilwurden, sollen hier nur einige Erwähnung finden:

- Förderungspreis der Stadt Wien

- Kardinal-Innitzer-Preis

- Ehrenpräsident der «Internationalen Gesellschaft für Chemo- und Immunotherapie»

- Mitglied der «Deutschen Akademie für Naturforscher Leopoldina»

- Ehrenmitglied der Österreichischen, Tschechischen, Ungarischen und Rumänischen Gesellschaften für Hämatologie

- Ehrenmitglied der «Gesellschaft der Ärzte in Wien»

- Ehrendoktorat der Schlesischen Medizinischen Akademie

- Ehrenpräsident der GAMED

Stacher war ein wertvoller und liebenswerter Mensch. Seine zutiefst soziale Gesinnung war kein Lippenbekenntnis, sondern ein Kennzeichen seiner Lebensführung. Was an ihm sterblich war, wurde am 19.08.2013 am Wiener Zentralfriedhof in seinem einfachen Familiengrab beigesetzt und dabei Abschied von ihm genommen. Sein Andenken werden wirdadurch ehren, dass wir sein letztes großes Werk, die GAMED, in seinem Sinne fortführen werden.

Wir werden ihn nie vergessen.

Wolfgang Marktl, Wien

Nachfolger von Alois Stacher als Präsident der Wiener Internationalen Akademie für Ganzheitsmedizin 